\title{
Advances in Materials
}

\section{Characterization of A Novel FR4/AIN Printed Circuit Board of High Thermal Conductivity}

\author{
Diancheng Qin, Yonglong Xiao, Kewei Liang \\ Guangdong LED Packaging-Used Heat Dissipation Substrate Engineering Technology Research Center, Rayben Technologies (Zhuhai) \\ Limited, Zhuhai, China
}

Email address:

mike_qin@rayben.com (Diancheng Qin)

\section{To cite this article:}

Diancheng Qin, Yonglong Xiao, Kewei Liang. Characterization of A Novel FR4/AlN Printed Circuit Board of High Thermal Conductivity. Advances in Materials. Vol. 7, No. 2, 2018, pp. 26-33. doi: 10.11648/j.am.20180702.13

Received: May 14, 2018; Accepted: June 6, 2018; Published: June 29, 2018

\begin{abstract}
High thermally conductive cuboid aluminum nitride block was used as reinforcement material to fill the FR4 matrix for the fabrication of a novel FR4/AIN PCB (Printed Circuit Board) with high thermal conductivity. This novel PCB was subjected to a thermal shock test of 1500 cycles from $-40^{\circ} \mathrm{C}$ keeping 30 minutes to $125^{\circ} \mathrm{C}$ keeping 30 minutes during 1000 hours with transfer time less than 10 seconds, presented an excellent reliability since there was no crack and delamination emerging. By performing a comparative study between FR4/AIN PCB and anodized MCPCB, it was found that the thermal resistance of both PCB were $0.63^{\circ} \mathrm{C} / \mathrm{W}$ and $2.74^{\circ} \mathrm{C} / \mathrm{W}$ respectively. When CREE XTE LEDs were mounted on FR4/AIN PCB and anodized MCPCB using SMT technology to dissipate heat respectively, the LEDs' junction temperature were $37^{\circ} \mathrm{C}$ and $42.1{ }^{\circ} \mathrm{C}$ and the overall corresponding thermal resistance were $3.93^{\circ} \mathrm{C} / \mathrm{W}$ and $6.43^{\circ} \mathrm{C} / \mathrm{W}$ accordingly. Therefore, a conclusion can be drawn that this novel PCB exhibits a more excellent heat dissipation performance than $30 \mathrm{~W} / \mathrm{m} \cdot \mathrm{K}$ anodized MCPCB does and it is a promising alternative of MCPCB for heat dissipation of high power electronic devices like LEDs.
\end{abstract}

Keywords: FR4/AIN PCB, Anodized PCB, Heat Dissipation, LED, Junction Temperature, Thermal Resistance

\section{Introduction}

Since the perpetual miniaturization, power increase and enhanced integration level of LEDs have generated serious heat accumulation that would adversely influence their comprehensive properties such as lifespan, reliability, luminous efficiency, etc [1-2]. it is essential to devise highly efficient and proper thermal management to dissipate the considerable amount of heat produced during their operation for improved and prolonged performance.

As a matter of fact, it is well acknowledged that PCBs of great heat dissipation performance act as an indispensable part and play a vital important role in LEDs' thermal management. PCBs possessing high thermal conductivity and reliability can contribute a lot to LEDs' practical engineering application. Generally, MCPCB (Metal Core Printed Circuit Board) and high thermally conductive ceramic are the most commonly used heat dissipation PCB materials. However, regular MCPCBs are usually of relatively low thermal conductivity due to their dielectric layer material, a composite employing thermal conductive fillers like $\mathrm{AlN}, \mathrm{Al}_{2} \mathrm{O}_{3}, \mathrm{BN}, \mathrm{Si}_{3} \mathrm{~N}_{4}, \mathrm{Cu}, \mathrm{Al}$,
Ag, MWCNT and graphene particles, etc. to fill polymer matrix to achieve a higher thermal conductivity [3-5]. But unfortunately, this type of composites, although presenting higher thermal conductivity than their matrix do, are still relatively low and can't meet high power LEDs' heat dissipation requirement [6-7]. As for ceramics, although they have always been experimentally reported to be high thermally conductive and are expected to be another promising heat dissipation alternative materials, their easy friability, high production cost and metallization difficulty pose a big challenge for their widespread application [8-9].

In present days, in order to enhance the thermal conductivity of MCPCB, a novel technology of forming a thick alumina film of high thermal conductivity using anodic oxidation on aluminium base has been proposed to replace its polymer/filler composited dielectric layer [10]. With the help of PVD and electroplating, a thick layer of copper would be obtained on alumina film. Thus, the aluminium base, alumina film and copper layer could be integrated into a 
novel anodized MCPCB of much higher thermal conductivity than that of regular MCPCB. However, except for its relatively higher thermal conductivity, this novel MCPCB are high cost, which therefore limits its application [11-12].

In this paper, a cost down FR4/AIN PCB with excellent heat dissipation performance was proposed. It was fabricated using cuboid aluminum nitride block as reinforcement to fill FR4 matrix which consists of copper, resin and reinforcement material [13]. High power electronic components like LEDs are supposed to be placed on aluminum nitride block to achieve highly efficient heat dissipation. And the FR4 part only serves to realize electrical connection and good mechanical processability. Additionally, a comparative study between FR4/AIN PCB and $30 \mathrm{~W} / \mathrm{m} \cdot \mathrm{K}$ anodized MCPCB was carried out to evaluate the superiority of FR4/AIN PCB for its heat dissipation performance and to seek an alternative to replace MCPCB for LEDs' thermal management.

\section{Experimental Section}

\subsection{FR4/AlN PCB Fabrication}

Table 1 illustrates the stackup of FR4/AIN PCB with a total thickness of $0.764 \pm 10 \% \mathrm{~mm}$. In this study, $7 \times 7 \times 0.635 \mathrm{~mm}$ thickness aluminum nitride block was required to be metalized $0.07 \mathrm{~mm}$ copper on top and bottom sides. Additionally, FR4 materials of S1000-2 CCL (Copper Clad Laminate) and S1000-2B prepreg were also demanded. CCL and prepreg were pre-routed a square hole through the perpendicular panel for the cuboid aluminum nitride block to embedded in. According to the stackup, the integration of FR4 materials and aluminum nitride block was achieved employing lamination technology as illustrated in Figure 1. Since lamination was carried out in need of certain temperature and pressure to enable prepreg soften and flow to fill the gap between FR4 materials and metalized aluminum nitride block, the prepreg was inevitably flow onto the surface of FR4/AIN PCB, so as illustrated in figure 2, the excessive prepreg on PCB's surface must be removed by scrubbing.

Table 1. The structure and stack up of FR4/AIN PCB.

\begin{tabular}{llllll}
\hline ID & Name & Supplier & Type & Description & Thickness \\
\hline 1 & CCL & ShengYi & S1000 & Copper & $0.07 \mathrm{~mm}$ \\
2 & PP & ShengYi & S1000B & Prepreg & $0.23 \mathrm{~mm}$ \\
3 & PP & ShengYi & S1000B & Prepreg & $0.082 \mathrm{~mm}$ \\
4 & CCL & ShengYi & S1000 & Core & $0.082 \mathrm{~mm}$ \\
\end{tabular}

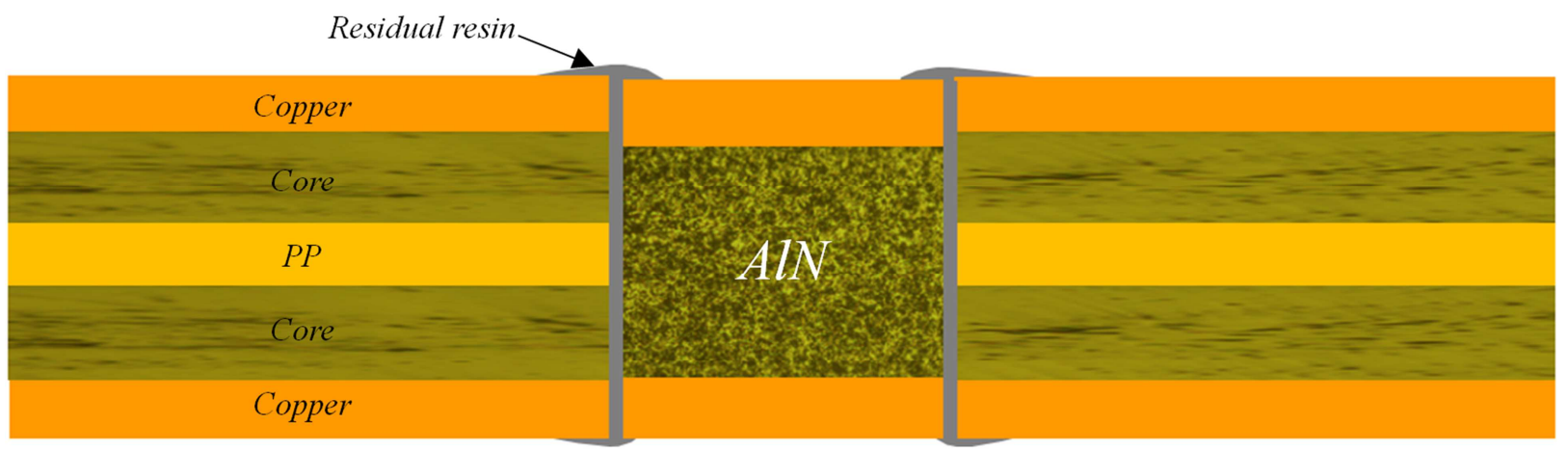

Figure 1. Schematic illustration of finished FR4/AIN PCB after lamination with residue resin on its surfaces.

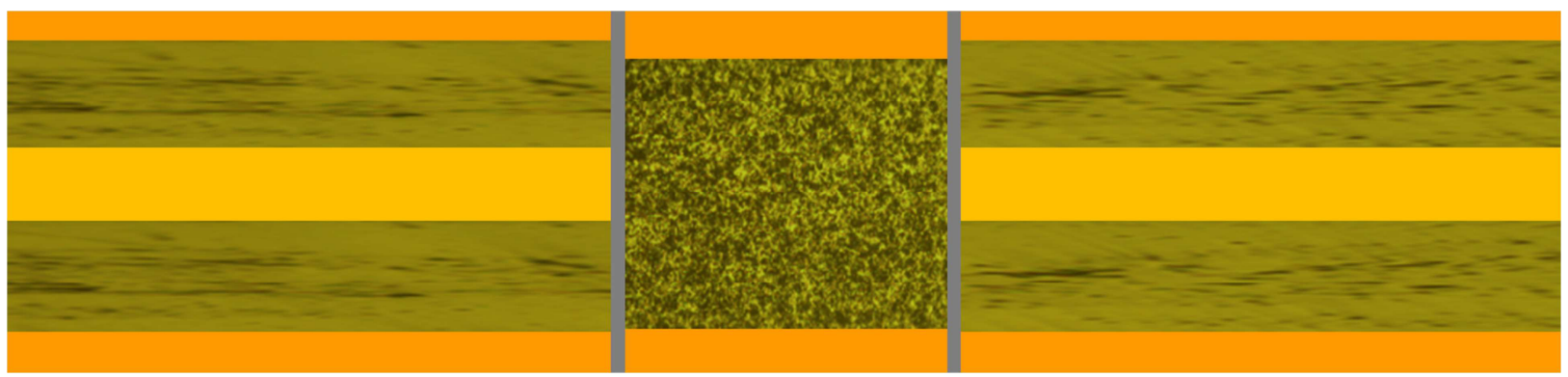

Figure 2. Schematic illustration of finished FR4/AIN PCB after lamination with residue resin removed.

\subsection{FR4/AIN PCB Characterization}

\subsubsection{Reliability}

A thermal shock test employing TS300 Temperature Shock
Test Chamber was performed to investigate the reliability of FR4-AIN interface under harsh environment. FR4/AIN PCB was subjected to 1500 cycles thermal shock during 1500 hours. The temperature started from $-40^{\circ} \mathrm{C}$, keeping 30 minutes, and 
then rising to $125^{\circ} \mathrm{C}$ within 10 seconds, keeping for 30 minutes as well.

When the thermal shock test was completed, the vertical micro-section of FR4/AIN interface was viewed using optical microscope to observe if there was any emergence of crack or delamination during the thermal shock process.

\subsubsection{Heat Dissipation Performance}

In order to characterize the heat dissipation performance of FR4/AIN PCB, XTE LEDs provided by CREE were mounted on FR4/AIN substrate and anodized MCPCB, forming modules called sample $\mathrm{A}$ and sample $\mathrm{B}$ respectively, employing SMT technology in the first place. Subsequently, According to JESD 51-1 and JESD 51-14 standards, the LEDs' junction temperature, thermal resistance of FR4/AIN substrate and anodized MCPCB as well as their corresponding modules were measured using T3Ster with the ambient temperature at $20^{\circ} \mathrm{C}$ and relative humidity at $49 \%$. Note that both FR4/AIN substrate and anodized MCPCB were the same thickness overall thickness of $0.764 \mathrm{~mm}$. the FR4 materials and cuboid aluminum nitride block possess thermal conductivity approximately $0.22 \mathrm{~W} / \mathrm{m} \cdot \mathrm{K}$ and $170 \mathrm{~W} / \mathrm{m} \cdot \mathrm{K}$ respectively. The anodized MCPCB boasts thermal conductivity up to 30 $\mathrm{W} / \mathrm{m} \cdot \mathrm{K}$.

\section{i. K Factor Measurement}

In view of forward voltage $\left(\mathrm{V}_{\mathrm{F}}\right)$ of LED's PN junction is extremely sensitive to temperature, there is a certain relationship between $\mathrm{V}_{\mathrm{F}}$ and temperature variation. If LED's self-heating can be neglected, assuming LED is placed in constant temperature chamber and this temperature is controllable, when temperature is changed and the $V_{F}$ is recorded simultaneously, then a straight line which reflects the relationship between $\mathrm{V}_{\mathrm{F}}$ and temperature variation will be resulted, and its gradient is called $\mathrm{K}$ factor.

In order to obtain LEDs' $T_{j}$ variation, the thermal grease was coated on the bottom of the samples to fix their temperature at $25 \pm 1{ }^{\circ} \mathrm{C}$ for measurement of $\mathrm{K}$ factors as shown in figure 3. During the following process, the junction temperature $\left(T_{j}\right)$ variation was determined using $V_{F}$ variation multiplied by $\mathrm{K}$ factor.

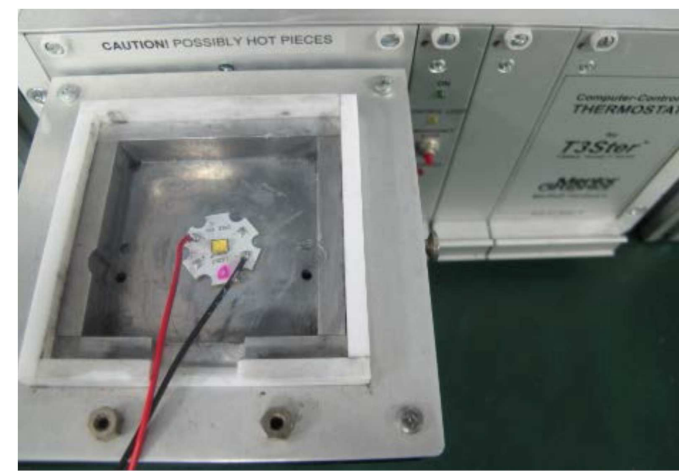

Figure 3. K factor measurement.

\section{ii. Optical Power Measurement}

In this part, TERALED was used to measure LED's total power (electric power) and optical power. Considering LED's total power consists of optical power and thermal power, the thermal power which is used to calculate thermal resistance will be obtained when total power (electric power) and optical power are known.

The samples in this study were fixed on the surface of the thermostat of TERALED as shown in figure 4 then the optical power and electric power of LEDs were measured by the integrating sphere as shown in figure 5.



Figure 4. Sample on the thermostat.



Figure 5. Electric power and optical power measurement employing Integrating sphere of TERALED.

\section{iii. Thermal Resistance Measurement}

According to the Standard JESD51-14, the thermal resistance was measured by the dual interface test method. In this paper, grease and thermal pad were used to connected PCB bottom sides to thermostat, keeping their temperature constant at $25 \pm 1{ }^{\circ} \mathrm{C}$ shown in figure 6 and figure 7 . The test current was set $1 \mathrm{~A}$; heating time was set $60 \mathrm{~s}$ and cooling time was set $60 \mathrm{~s}$.



Figure 6. Interface of LED/thermostat with thermal grease. 




Figure 7. Interface of LED/thermostat with thermal pad.

\section{Results and Discussion}

\subsection{Bonding Strength of FR4/AlN Interface}

with the help of increased temperature and pressure during lamination, the resin was converted from solid state to viscous state. Simultaneously, the liquid resin began to flow and fill the gap between prepreg and aluminium nitride. Due to the viscidity of resin itself, the FR4 material and aluminium nitride achieved a combination. Figure 8 is the cross section view of resin/AIN PCB under optical microscope. There is no crack or delamination observed on resin/AIN interface after being subjected to 1500 cycle thermal shock from $-40^{\circ} \mathrm{C}$ to $125^{\circ} \mathrm{C}$. It can be deduced that the resin performed effective wettability to aluminium nitride surface to form an interface of strong bonding strength when the lamination process was applied.
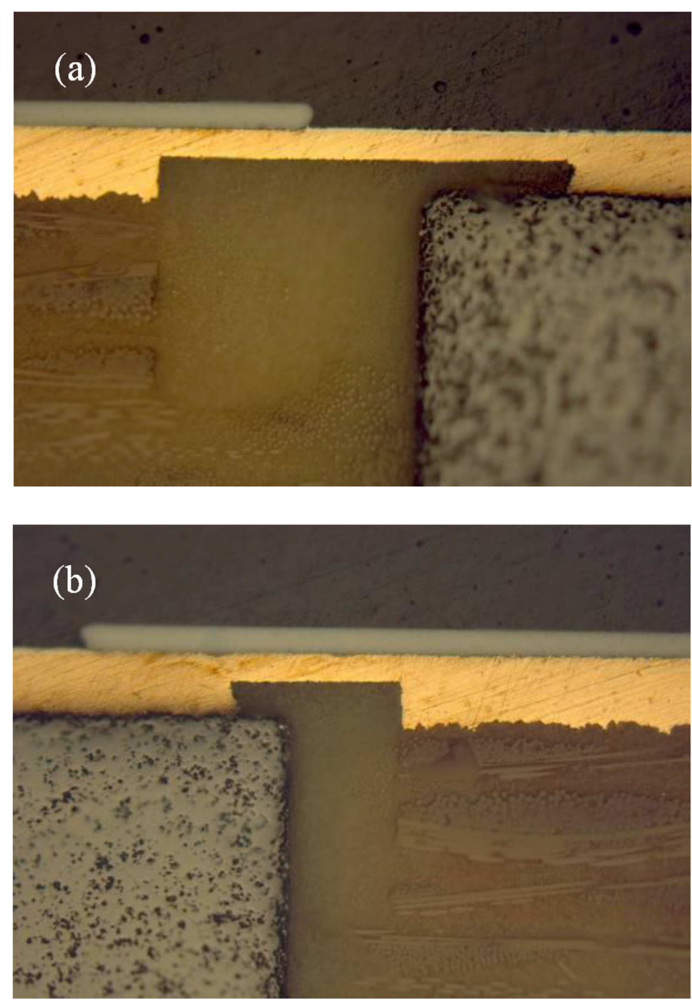

Figure 8. Cross section images of FR4/AlN Interface.

\subsection{Heat Dissipation Performance of FR4/AlN Substrate}

Figure 9 and figure 10 display the $\mathrm{K}$ factor curves of sample $\mathrm{A}$ and sample B. It is a reflection of the relation between LED's junction temperature $T_{j}$ and voltage. The gradient of the curve is $\mathrm{K}$ factor.

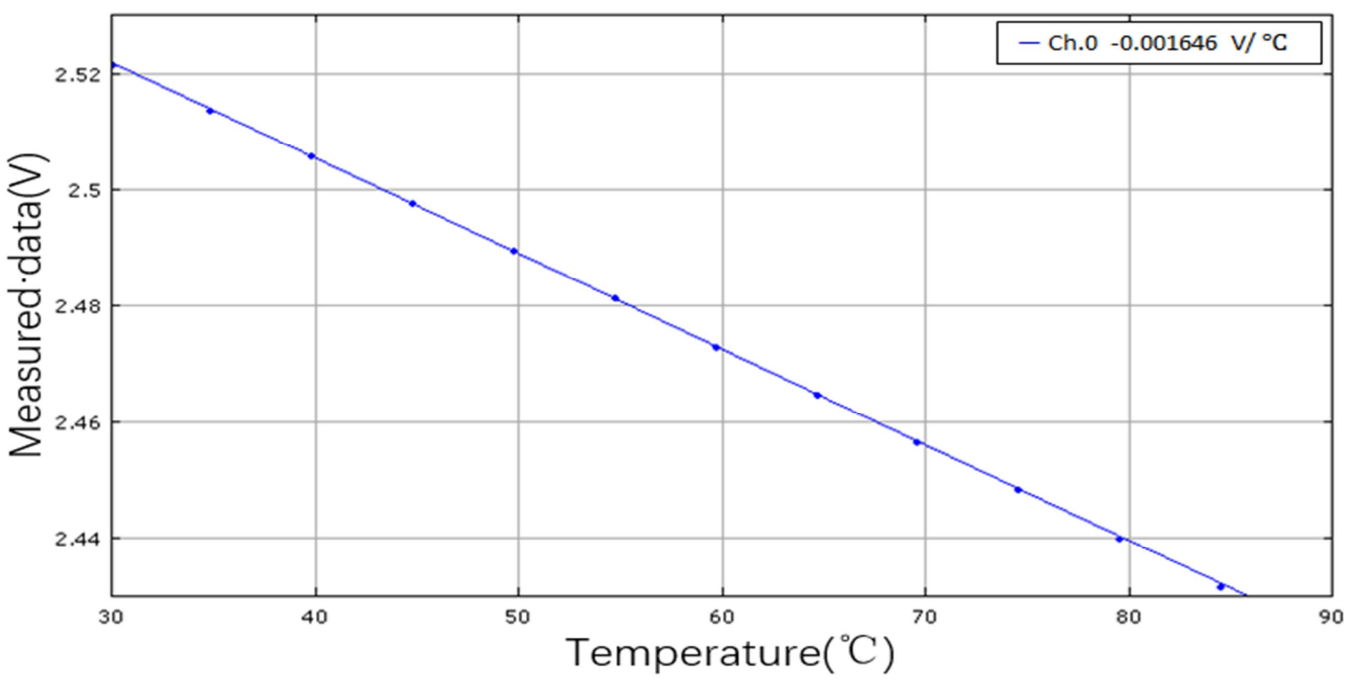

Figure 9. $K$ factor curve of sample A.

Generally, if $\mathrm{K}$ factor is decided, the $\mathrm{T}_{\mathrm{j}}$ variation can be calculated by the following equation [14]:

$$
\Delta \mathrm{T}=\Delta \mathrm{V}_{\mathrm{F}} \cdot \mathrm{K} \cdot
$$

Where $\Delta \mathrm{T}$ is the variation of $\mathrm{T}_{\mathrm{j}}, \Delta \mathrm{V}_{\mathrm{F}} \cdot$ is the voltage variation. 


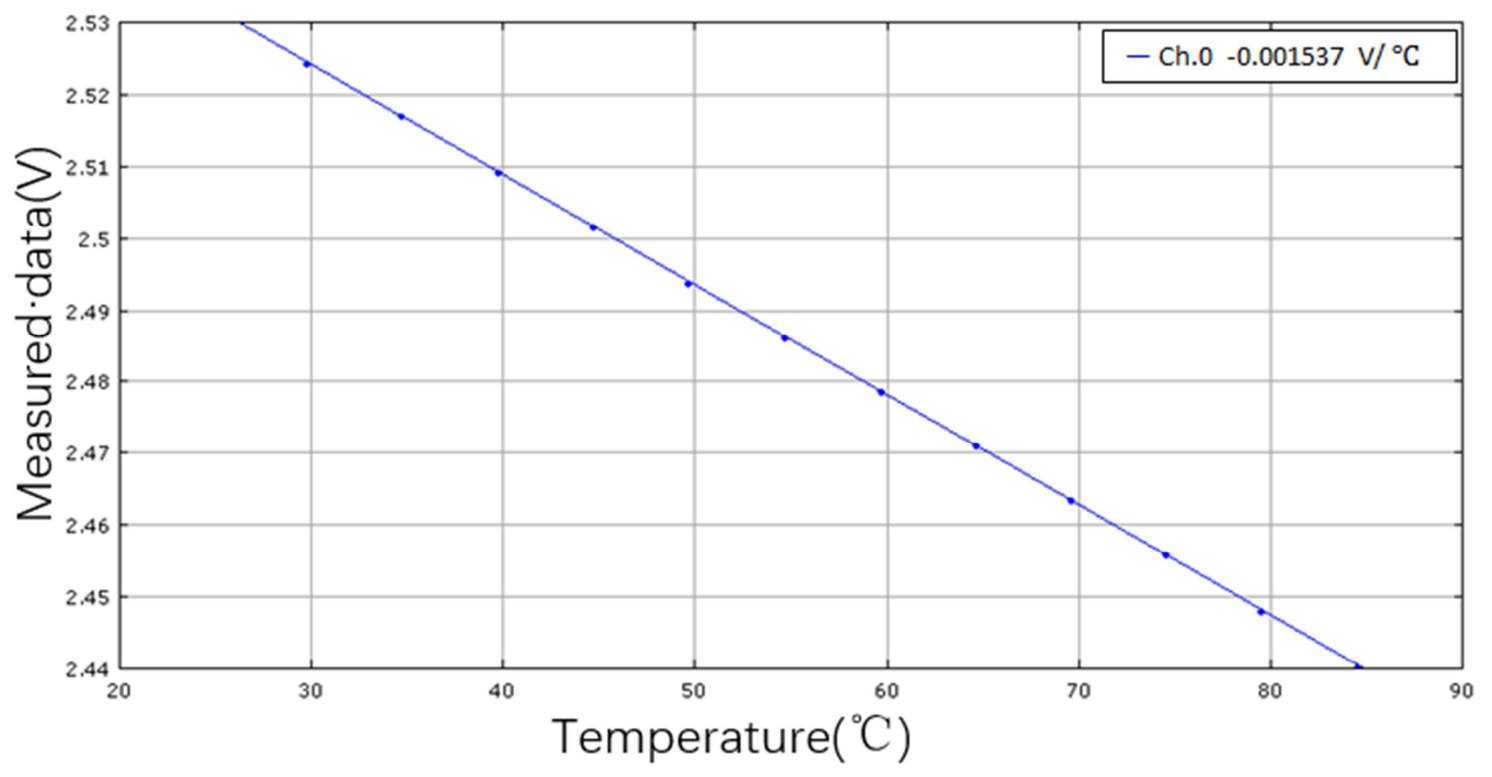

Figure 10. $K$ factor curve of sample B.

Figure 11 and Figure 12 show the Cooling curve of LED modules with the bottom side temperature of PCBs fixed at $25^{\circ} \mathrm{C}$. When LEDs were lighted, the $\mathrm{T}_{\mathrm{j}}$ increased as time elapses. When $\mathrm{T}_{\mathrm{j}}$ reached stabilization, a small current is given to keep LED's lighting and let $\mathrm{T}_{\mathrm{j}}$ decreased to another stabilization. During this process, the voltage variation was recorded. According to equation $1, \mathrm{~T}_{\mathrm{j}}$ variation $\Delta \mathrm{T}$ was obtained via voltage variation $\Delta \mathrm{V}_{\mathrm{F}}$. Since the temperature on the bottom side of FR4/AIN and is $25^{\circ} \mathrm{C}$ and the $\Delta \mathrm{T}$ is $12.04^{\circ} \mathrm{C}$, then the $\mathrm{T}_{\mathrm{j}}$ of sample A could be deduced $37.04^{\circ} \mathrm{C}$. Meanwhile, Since the temperature on the bottom side of anodized MCPCB was $25^{\circ} \mathrm{C}$ and the $\triangle \mathrm{T}$ was $17.1^{\circ} \mathrm{C}$, then the $\mathrm{T}_{\mathrm{j}}$ could be deduced $42.1^{\circ} \mathrm{C}$. so, it is obvious that the junction temperature of sample $\mathrm{A}$ is approximately $5^{\circ} \mathrm{C}$ lower than that of sample B, which reveals that FR4/AIN PCB demonstrates a much better heat dissipation performance than anodized MCPCB does.



Figure 11. Cooling curve of sample A with bottom side fixed at $25^{\circ} \mathrm{C}$. 




Figure 12. Cooling curve of sample B with bottom side fixed at $25^{\circ} \mathrm{C}$.

Figure 13 and figure 14 show LED modules' differential structure functions, from which it is easy to obtain the thermal resistance information of each layer of LED modules. In this paper, thermal grease and thermal pad were used as interfacial materials to bond LED module and thermostat, so there were two different thermal resistance curves in each sample's differential structure function. As shown in both Figures, the blue ones represent thermal resistance curves of modules using thermal grease, the red ones represent thermal resistance curves of modules using thermal pad. This is because thermal grease is more thermally conductive than thermal pad. The overall thermal resistance of the system is expected to be much higher when thermal pad is applied. Note that there is an overlapping part of both curves before a parting point's appearance. Based on the aforementioned analysis, it can be easily understood that from zero to parting point, the thermal resistance of each layer of sample A and B are indicated.

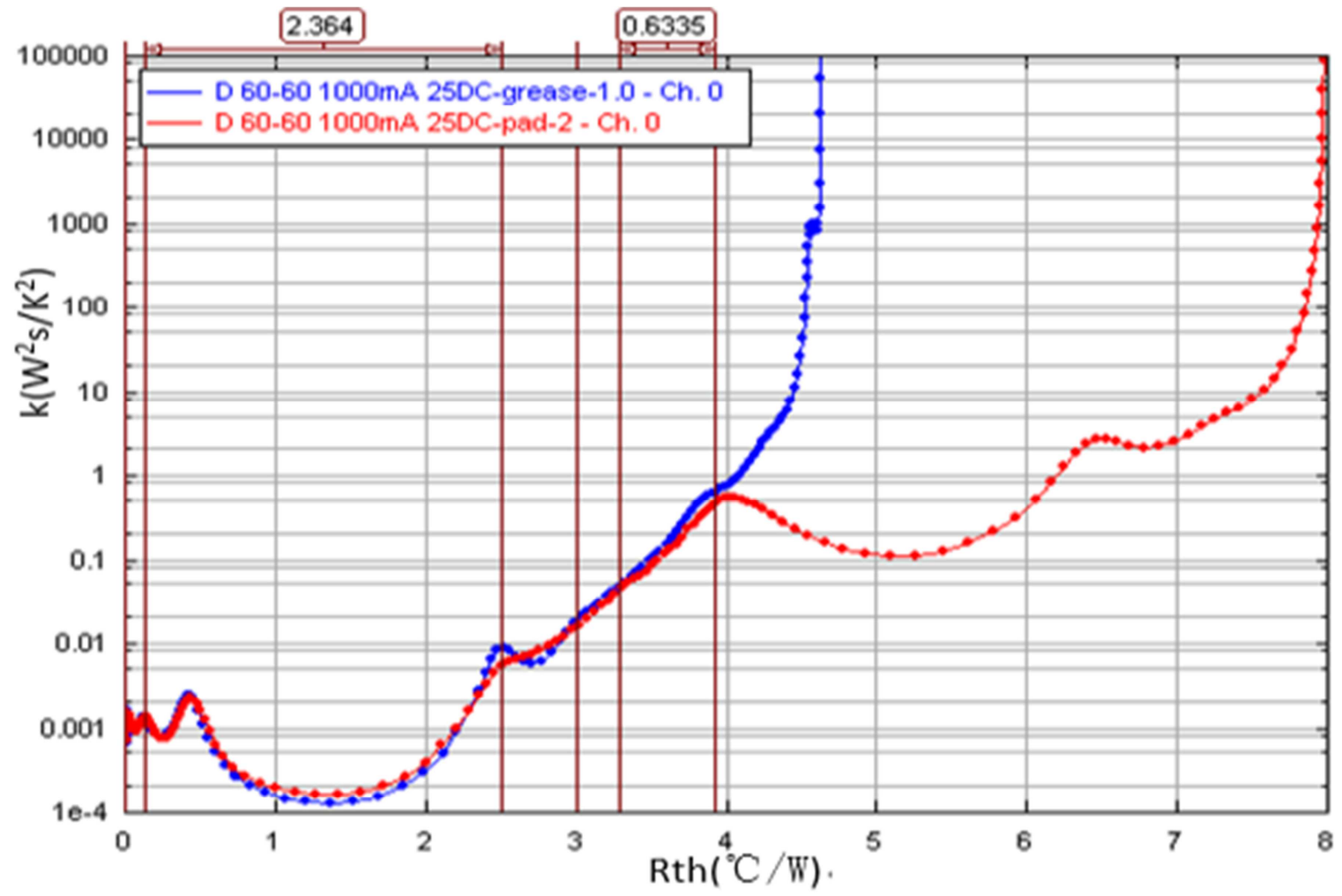

Figure 13. Differential structure function of sample A. 




Figure 14. Differential structure function of sample $B$.

Table 2. Thermal Characteristic of sample A and sample B.

\begin{tabular}{lllllll}
\hline Sample & $\begin{array}{l}\text { Electrical Power } \\
(\mathbf{W})\end{array}$ & $\begin{array}{l}\text { Optical Power } \\
(\mathbf{W})\end{array}$ & $\begin{array}{l}\text { Heat Power } \\
(\mathbf{W})\end{array}$ & $\begin{array}{l}\mathbf{K} \text { factor } \\
\left(\mathbf{m V} /{ }^{\circ} \mathbf{C}\right)\end{array}$ & $\begin{array}{l}\text { Total Thermal Resistance }\left({ }^{\circ} \mathbf{C}\right. \\
/ \mathbf{W})\end{array}$ & $\begin{array}{l}\text { Junction Temperature } \\
\left({ }^{\circ} \mathbf{C}\right)\end{array}$ \\
\hline $\mathrm{A}$ & 3.562 & 1.024 & 2.538 & -1.646 & 3.93 & 37 \\
$\mathrm{~B}$ & 3.475 & 1.022 & 2.453 & -1.503 & 6.43 & 42.1 \\
\hline
\end{tabular}

Table 3. Thermal Resistance of sample $A$ and sample $B\left({ }^{\circ} \mathrm{C} / W\right)$.

\begin{tabular}{lllllll}
\hline Sample & Chip & Die attach & Substrate & Solder & PCB & Total \\
\hline A & 0.14 & 2.36 & 0.51 & 0.28 & 0.63 & 3.93 \\
B & 0.13 & 2.43 & 0.69 & 0.43 & 2.74 & 6.43 \\
\hline
\end{tabular}

Table 3 and table 4 displays the thermal characteristic and thermal Resistance of both samples. As demonstrated in table 2, LED's junction temperature of sample A is $37^{\circ} \mathrm{C}$ and LED's junction temperature of sample $\mathrm{B} 42.1^{\circ} \mathrm{C}$, which suggests FR4/AlN PCB performs much better than anodized MCPCB to dissipate the heat produced during LED's operation. In table 3, it is clearly to find out that the thermal resistance of FR4/AlN PCB is $0.63^{\circ} \mathrm{C} / \mathrm{W}$, much lower than $2.74^{\circ} \mathrm{C} / \mathrm{W}$ of anodized MCPCB, which further proves FR4/AIN PCB is high thermally conductive than anodized MCPCB.

To facilitate the analysis of thermal conductivity superiority FR4/AIN PCB over anodized MCPCB, some basic concepts are supposed to be introduced. It has been proved that heat conduction in metallic materials and inorganic materials majorly depends on Phonon propagation and free electron movement [15-16]. In metallic materials, free electron movement dominates the heat conduction process. However, it is the phonon propagation that determines the heat conduction process in inorganic materials. In the present study, aluminium nitride block was used as reinforcement to fill the resin matrix. Since aluminium nitride and resin both are inorganic materials, phonon propagation is the major way to dissipate heat. Note that aluminium nitride and resin have totally different crystal lattice. Additionally, aluminium nitride possesses an $170 \mathrm{~W} / \mathrm{m} \cdot \mathrm{K}$ thermal conductivity, very much higher than 0.22 $\mathrm{W} / \mathrm{m} \cdot \mathrm{K}$ of resin. When phonons cross the resin/AlN interface, a scattering process occurs due to the different crystal lattice between aluminium nitride and resin, which means only part of phonons would successfully spread into resin side from aluminium nitride side. Hence, the interface thermal resistance or called Kapitza thermal resistance will be resulted [17-19]. At the same time, since the thermal conductivity difference between them is huge, the major part 
of heat will be transmitted through aluminium nitride ceramic from its top to its bottom side. In other words, aluminium nitride ceramic acted as the major heat conduction pathway in resin/AlN PCB, which well explained its excellent heat dissipation performance in sample A.

As far as anodized MCPCB is concerned, the heat produced by LED during operation must conducted from anodic oxide film to aluminium base. Since anodic oxide film solely depends on phonon propagation for heat conduction and aluminium base majorly depends on free electron movement for heat conduction, there was a very high Kapitza thermal resistance emerging on their interface. Which greatly increased anodized MCPCB's overall thermal resistance along its perpendicular direction.

\section{Conclusions}

Resin/AlN PCB was obtained using aluminium nitride block as reinforcement to fill resin matrix and employing lamination technology to complete combination. The Resin/AIN interface, which was subjected to 1500 cycles thermal shock from $-40^{\circ} \mathrm{C}$ to $125^{\circ} \mathrm{C}$, exhibited an excellent bonding strength due to the good Wetting ability of resin to aluminium nitride surface since there was no crack or delamination occurrence. When compared with anodized $\mathrm{MCPCB}$ of $30 \mathrm{~W} / \mathrm{m} \cdot \mathrm{K}$ conductivity, the Resin/AlN PCB displayed a evidently better heat dissipation performance with the LED junction temperature and thermal resistance lower by approximately $5^{\circ} \mathrm{C}$ and $2.11^{\circ} \mathrm{C} / \mathrm{W}$ respectively. This is mainly because the Kapitza thermal resistance of Resin/AIN PCB present in horizontal plane, which confined the great part of heat in aluminium nitride ceramic for efficient dissipation due to the ceramic's high thermal conductivity up to $170 \mathrm{~W} / \mathrm{m} \cdot \mathrm{K}$. while the Kapitza thermal resistance of anodized MCPCB present in perpendicular plane hindering heat flux transportation, which added to its overall thermal resistance. Considering high thermal dissipation performance of FR4/AIN PCB, it is expected to be a promising heat dissipation material for high power electronic devices' thermal management.

\section{References}

[1] Yong $\mathrm{Hu}$, Guoping Du, Nan Chen (2016). A novel approach for $\mathrm{Al}_{2} \mathrm{O}_{3} /$ resin composites with high strength and thermal conductivity. Composites Science and Technology 124, 36-43.

[2] M. Wasim Akhtar, Yun Seon Lee, Dong Jin Yoo and Jong Seok Kim (2017). Alumina-graphene hybrid filled resin composite: Quantitative validation and enhanced thermal conductivity. Composites Part B 131, 184-195.

[3] Wenhui Yuan, Qiangqiang Xiao and Li Li (2016). Thermal conductivity of resin adhesive enhanced by hybrid graphene oxide/AlN particles. Applied Thermal Engineering 106, 1067-1074.

[4] Seran Choi, Jooheon Kim (2013). Thermal conductivity of resin composites with a binary-particle system of aluminum oxide and aluminum nitride fillers. Composites: Part B 51, $140-147$.

[5] Osman Eksik, Stephen F. Bartolucci and Tushar Gupta (2016). A novel approach to enhance the thermal conductivity of resin nanocomposites using graphene core shell additives. Carbon $101,239-244$.

[6] So Youn Mun, Hyung Mi Lim and Seung-Ho Lee (2018). Thermal and electrical properties of resin composite with expanded graphite-ceramic core-shell hybrids. Materials Research Bulletin 97, 19-23.

[7] Zhifang Gao and Lei Zhao (2015). Effect of nano-fillers on the thermal conductivity of resin composites with micro-A12O3 particles. Materials and Design 66, 176-182.

[8] Mehdi Derradji, Xuemei Song and Abdul Qadeer Dayo (2017) Applied Thermal Engineering. Applied Thermal Engineering $115,630-636$.

[9] QIN Diancheng, LI Baozhong and XIAO Yonglong (2017). Current Status and Development of Ceramic Metallization. China Ceramic Industry 24 (05), 30-36.

[10] QIN Dian-cheng, CHEN Ai-bing and XIAO Yong-long (2018) Preparation of Anodic Alumina Substrate and Its Application in LED Heat Dissipation. Journal of Synthetic Crystals 47 (01), 225-230.

[11] SUN Yan-le, XUAN Tian-peng and XU Shao-nan (2010). Research Advances on Anodic Oxidation of Aluminum Alloy. Plating and Finishing 32 (04), 18-21.

[12] ZHOU Ke-ke, HUANG Yan-bin and SANG Hao-ran (2016). Effect of Aluminum Alloy Anodic Oxidation Film Structure on the Adhe-sive Property. Surface Technology 45 (09), 188-193.

[13] Qin Diancheng, Li Baozhong and Huang Yizhao (2017). Interface Morphology and Thermal Conductivity of Ceramic-Embedded FR4 Structure. semiconductor technology 42 (11), 864-869.

[14] S. F. Sufian, M. Z. Abdullah (2017). Heat transfer enhancement of LEDs with a combination of piezoelectric fans and a heat sink. Microelectronics Reliability 68, 39-50.

[15] Thi My Linh Dang, Chang-Yeoul Kim and Yaming Zhang (2017). Enhanced thermal conductivity of polymer composites via hybrid fillers of anisotropic aluminum nitride whiskers and isotropic spheres. Composites Part B 114, 237-246.

[16] Yuan-Xiang Fu, Zhuo-Xian He and Dong-Chuan Mo (2014). Thermal conductivity enhancement of resin adhesive using graphene sheets as additives. International Journal of Thermal Sciences 86, 276-283.

[17] Liuyan Yin, Xingui Zhou and Jinshan Yu (2016). Fabrication of a polymer composite with high thermal conductivity based on sintered silicon nitride foam. Composites: Part A 90, 626-632.

[18] Shi-hui Song, Hideyuki Katagi, Yoshitaka Takezawa (2012). Study on high thermal conductivity of mesogenic resin resin with spherulite structure. Polymer 53, 4489-4492.

[19] Hyungu Im and Jooheon Kim (2012). Thermal conductivity of a graphene oxide-carbon nanotube hybrid/resin composite. CARBON 50, 5429-5440. 